\title{
Combined Technique for the Application of Micropulse Cyclophotocoagulation in Patients with Uncontrolled Glaucoma: Cyclo Mix
}

\author{
Loayza-Gamboa Waldo ${ }^{1}$, Herrera-Quiroz Julio ${ }^{2}$, Chávez-Vallejos Jennifer ${ }^{3}$, Valderrama-Albino Vanessa ${ }^{4}$, \\ Córdova-Crisanto Luis ${ }^{5}$, Alvarado-Villacorta Rosa ${ }^{6}$, Valera-Cornejo Diego Alejandro ${ }^{7}$
}

\begin{abstract}
Purpose: To describe the outcomes of a combined technique (Cyclo Mix) in uncontrolled glaucoma cases.

Materials and methods: Retrospective study. The Supra $810 \mathrm{~nm}$ subliminal laser (Quantel Medical, Cournon d'Auvergne, France) was used. A combined technique was performed using the subliminal mode (Subcyclo) in one hemifield (power of 2,000 mW, a duty cycle of 35\%, and 80-150 seconds), and the continuous wave mode (Thermo Cyclo) on the other hemifield (power of 1,000 mW, exposure time of 2 seconds per spot). The primary endpoint was the probability of surgical failure. Mean intraocular pressure (IOP) change, best-corrected visual acuity (BCVA), number of glaucoma eye drops, and complications at 6 months postoperatively were secondary outcomes.

Results: Twenty-three eyes from 13 patients were included. Mean age was $61.4 \pm 16.7$ (range: 18-78 years), and $69 \%$ were female, with a baseline IOP of $20.3 \pm 5.9$ (range: $13-38 \mathrm{~mm} \mathrm{Hg}$ ). The cumulative probability of failure was 18 and $22 \%$ on days 90 and 180, respectively. Mean IOP reduction was $-25.6 \pm 20.9 \%$ at 6 months. The number of glaucoma eye drops was reduced to $1.2 \pm 1(p=0.0024)$ at 6 months. No statistically significant change in the mean BCVA compared with baseline at 6 months was found $(p=0.84)$, and no severe complications were reported. Conclusion: Cyclo Mix seems to be a safe therapy that effectively reduces the IOP and glaucoma medications in eyes with uncontrolled openangle glaucoma for up to 6 months.

Keywords: Cyclodestruction, Glaucoma, Intraocular pressure, Micropulse laser, Transscleral cyclophotocoagulation.

Journal of Current Glaucoma Practice (2020): 10.5005/jp-journals-10078-1289
\end{abstract}

\section{INTRODUCTION}

Glaucoma is considered one of the leading causes of irreversible blindness globally and is defined as progressive optic neuropathy, which is strongly correlated with elevated intraocular pressure (IOP) for its progression. ${ }^{1,2}$ Controlling or lowering the IOP is the aim of the treatment to preserve vision. ${ }^{3}$ Many strategies have been used, such as topical medications, laser therapy, or surgical treatment, all of them have different mechanisms of action (increasing outflow of aqueous humor, decreasing its production, or both) that finally reduce the IOP. ${ }^{4}$ Cyclodestruction of the ciliary body was used since 1930 with diathermy or cryotherapy to decrease the production of aqueous humor and thus reduce IOP. ${ }^{5}$ Thirty years later, laser cyclophotocoagulation (CPC) was introduced; thereafter, the diode laser was begun to be studied, ${ }^{6}$ and finally, in 1992, the transscleral (TS) diode laser CPC was reported to reduce IOP successfully. ${ }^{7}$ However, this procedure has been associated with severe visual complications and was used for several years in patients with advanced refractory glaucoma. ${ }^{8}$ These complications are thought to manifest the excess of energy produced by the continuous wave of the diode laser therapy, which would damage the surrounding tissue (ciliary body, stroma, ciliary muscles, and non-pigmented epithelium). ${ }^{9}$ In the last years, micropulse (MP) technology has gained more importance; this strategy breaks the continuous wave laser into multiple short and repetitive pulses that allow the tissue to cool down between applications, thus reducing thermal damage. This strategy delivers very short energy pulses followed by rest periods, which is known as "duty cycle" and is defined as the ratio of time that a laser is delivering energy vs resting (ON/OFF), therefore,
1,3Glaucoma Department, Peruvian National Eye Institute "Francisco Contreras Campos", Lima, Perú; Research Department, Clínica Vista, Lima, Peru; Cayetano Heredia Peruvian University, Lima, Peru

2,4,6,7 Research Department, Clínica Vista, Lima, Peru; Cayetano Heredia Peruvian University, Lima, Peru

${ }^{5}$ Research Department, Clinica Vista, Lima, Peru; National University of San Marcos, Lima, Peru

Corresponding Author: Valera-Cornejo Diego Alejandro, Research Department, Clínica Vista, Lima, Peru; Cayetano Heredia Peruvian University, Lima, Peru, Phone: +51987017264, e-mail: diegoavalera@ gmail.com

How to cite this article: Waldo $L-G$, Julio $H-Q$, Jennifer $C-V$, et al. Combined Technique for the Application of Micropulse Cyclophotocoagulation in Patients with Uncontrolled Glaucoma: Cyclo Mix. J Curr Glaucoma Pract 2020;14(3):93-97.

Source of support: Nil

Conflict of interest: None

it does not reach the coagulative threshold, reducing collateral tissue damage. ${ }^{10}$ Micropulse transscleral cyclophotocoagulation (MP-TSCFC), which is a variation of conventional continuous-wave CFC, delivers energy using the micropulse strategy, and it has emerged as an attractive alternative for the treatment of many types of glaucoma, and more studies are reporting promising results in terms of efficacy and safety. ${ }^{11-15}$ Even though this procedure is also effective in cases with good visual acuity, ${ }^{12}$ many subtypes of glaucoma will not respond adequately to this therapy and will require additional treatment. Our goal was to describe the 
clinical outcomes of a modified technique (Cyclo Mix) in cases with uncontrolled glaucoma.

\section{Materials and Methods}

\section{Study Design}

This was a retrospective analysis of medical records from all patients who received the modified MP TSCPC from November 2018 through August 2019 at the glaucoma Department of the National Eye Institute of Peru. Ethical approval was obtained from the Review Board of the Institute (RCEI-56) which complied with the principles of the Declaration of Helsinki. Written informed consent was obtained previously to the procedure.

\section{Participants}

Twenty-three eyes from 13 patients who underwent modified MP-CPC were analyzed. Inclusion criteria consisted of patients with uncontrolled glaucoma (IOP levels above the target threshold or progression of the disease despite maximal medical therapy), initial best-corrected visual acuity (BCVA) worse than 20/25, and at least 6 months of follow-up. Most of the patients had failed or were unsuitable for conventional glaucoma surgery (filtering surgery or drainage device implant). Patients younger than 18 years, those with previous intraocular surgery, laser treatment, or any CPC within 6 months of the procedure were excluded.

\section{Subjects, Follow-up, and Measure the Outcome}

Baseline demographic and clinical characteristics such as sex, age, glaucoma type, number of glaucoma eye drops, previous glaucoma surgery, preoperative baseline IOP [measured with Goldmann applanation tonometry (GAT) (Haag Streit, Koeniz, Switzerland)], and BCVA were registered. The follow-up analysis was made on days 1,7 , months 1,3 , and 6 postoperatively. At each visit, BCVA, IOP levels, anterior segment examination, and the number of glaucoma eye drops needed to control the IOP were recorded. The IOP level was calculated using the average of 2 measurements which were taken with a difference of 10 minutes. Best-corrected visual acuity data were turned into LogMAR (Logarithm of the Minimum Angle of Resolution) units for the quantitative analysis.

\section{Outcome Measures}

The probability of failure was our primary outcome. The definition of failure was any of the following: $<20 \%$ IOP reduction from baseline or an IOP that falls out of this range $(5-18 \mathrm{~mm} \mathrm{Hg}$ ) at two consecutive visits, additional glaucoma surgery, or loss of light perception vision. Criteria used to define complete success were the absence of failure without the use of glaucoma eye drops and visual loss of $<2$ lines (Snellen chart) and qualified success was the lack of failure with the use of glaucoma medications. ${ }^{16}$

The mean change on IOP, BCVA at 6 months after the procedure, number of glaucoma eye drops and complications such as uveitis, mydriasis, cataract, persistent hypotony, and phthisis were secondary outcomes.

\section{Surgical Procedure}

The treatment was performed under peribulbar anesthesia, administered with a combination (1:1) of bupivacaine $0.5 \%$ and lidocaine 2\%. The Supra $810 \mathrm{~nm}$ SubLiminal ${ }^{\circledR}$ (Quantel Medical, Cournon d'Auvergne, France) laser was used; the probe tip was placed $3 \mathrm{~mm}$ behind the limbus. Our technique (Cyclo Mix) used different strategies on each hemifield. Superior hemifield: The laser was set on subliminal mode (Subcyclo) using 2,000 mW of power, $35 \%$ of duty cycle, and was applied for a total of $80-150$ seconds on the superior perilimbal region (Meridians from 10 to 2 o'clock) with a continuous sliding motion; 3 and 9 o'clock meridians were avoided. Inferior hemifield: The laser was set on continuous wave mode (Thermo Cyclo) with the following parameters (Power: $1,000 \mathrm{~mW}$ with an exposure time of 2 seconds per spot) and the treatment was applied on the inferior perilimbal region (Meridians from 4 to 8 o'clock) avoiding the 3 and 9 o'clock meridians. The laser power was reduced by $250 \mathrm{~mW}$ if a "pop" sound was heard. The complete technique is illustrated in Figure 1. A subconjunctival triamcinolone acetonide (@Kenacort-A 40, Bristol-Myers Squibb, Australia) injection was performed on the perilimbal area $(2 \mathrm{mg} / 0.5$ $\mathrm{mL}$ ) at the end of the surgery. After that, an eye patch was placed for 12 hours, and topical moxifloxacin $0.5 \%$ with dexamethasone 0.1\% (@Vigadexa, Alcon Laboratories, Inc., Fort Worth, TX, USA) was started during the first week which was tapered in the second week. Additionally, patients were advised not to discontinue their previous anti-glaucomatous treatment.

\section{Statistical Analysis}

The database was evaluated for "missing values" and inconsistencies. The Shapiro-Wilk test was used to assess if data were normally distributed. Variables were reported using means \pm standard deviation (SD), and number ( $n$ ) and percentages (\%) for continuous and categorical variables, respectively. Wilcoxon test and paired $t$-test were used to compare groups, and Kaplan-Meier analysis to calculate the failure probability. @Stata software v.15.1 (StataCorp. 2017, Tx: StataCorp LLC) and GraphPad Prism software v.8.4.2 for Windows (GraphPad Software, San Diego, CA, USA) were used. A $5 \%$ level of significance was considered.

\section{Results}

Twenty-three eyes from 13 patients were included. The mean age was $61.4 \pm 16.7$ (range: $18-78$ years), and $69 \%$ were female. Primary open-angle glaucoma was the most frequent diagnosis, and $73.9 \%$ did not have previous glaucoma surgery. Mean baseline BCVA was $0.75 \pm 0.59$ LogMAR (range: $0.1-2.1$ ). Table 1 summarizes baseline characteristics. In Figure 2, the Kaplan-Meier plot depicts the cumulative survival. The cumulative probability of failure was 18 and $22 \%$ on days 90 and 180 , respectively. Complete success was achieved in $30.4 \%$ of cases, while qualified success (IOP criteria reduction with the additional use of topical medication) in $87 \%$ at the last follow-up. Intraocular pressure at the last follow-up was 14.4 \pm 3.9 (range: $9-27$ ) with a significant reduction ( $p<0.0001$ ) (Fig. 3); in addition, the mean percentage of IOP reduction was $-28.3 \pm 24.4$,

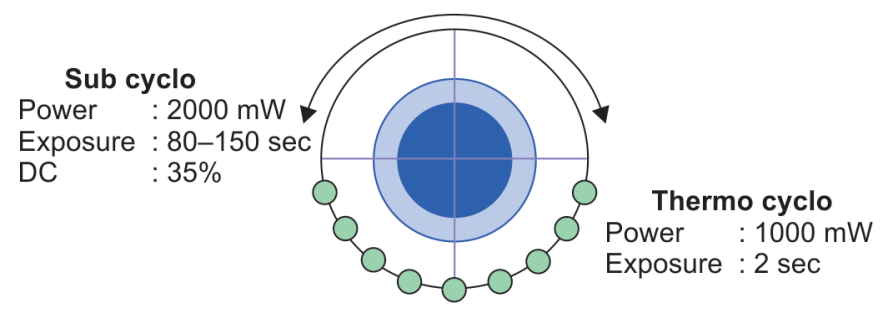

Fig. 1: Representative drawing demonstrating the Cyclo Mix technique. First, the probe is set on Micropulse mode (Sub Cyclo) and applied with a continuous movement on the superior hemifield for 8 seconds (Green arrows). After that, the probe is changed to continuous-wave mode (Thermo Cyclo) and applied with 10-20 shots over the inferior hemifield (red circles) 


\begin{tabular}{|c|c|}
\hline Variable & Value \\
\hline Age (years), $\bar{x} \pm \mathrm{SD}$ (range)* & $61.4 \pm 16.7(18-78)$ \\
\hline \multicolumn{2}{|l|}{ Sex, $n(\%)^{*}$} \\
\hline Female & $9(69.2)$ \\
\hline \multicolumn{2}{|l|}{ Diagnosis/etiology } \\
\hline Primary open-angle glaucoma & $12(52.2)$ \\
\hline Primary angle-closure glaucoma & $4(17.4)$ \\
\hline Pseudoexfoliation glaucoma & $2(8.7)$ \\
\hline Silicone oil-induced glaucoma & $2(8.7)$ \\
\hline Glaucoma after PK & $1(4.3)$ \\
\hline Congenital glaucoma & $2(8.7)$ \\
\hline \multicolumn{2}{|l|}{ Lens status, $n$ (\%) } \\
\hline Phakic & $9(39.1)$ \\
\hline Pseudophakia & $14(60.9)$ \\
\hline \multicolumn{2}{|l|}{ Prior glaucoma surgery, $n$ (\%) } \\
\hline None & $17(73.9)$ \\
\hline Trabeculectomy & $4(17.4)$ \\
\hline Tube shunt & $2(8.7)$ \\
\hline Cup to disk ratio, $\bar{x} \pm \mathrm{SD}$ (range) & $0.9 \pm 0.06(0.8-0.99)$ \\
\hline $\begin{array}{l}\text { Baseline number of glaucoma medications, } \\
\bar{x} \pm \mathrm{SD} \text { (range) }\end{array}$ & $2.7 \pm 1.5(0-4)$ \\
\hline Baseline BCVA, LogMAR, $\bar{x} \pm$ SD (range) & $0.75 \pm 0.59(0.1-2)$ \\
\hline Baseline IOP (mm Hg), $\bar{x} \pm \mathrm{SD}$ (range) & $20.3 \pm 5.9(13-38)$ \\
\hline
\end{tabular}

$\bar{x}$, mean; SD, standard deviation; $\mathrm{PK}$, penetrating keratoplasty; $\mathrm{BCVA}$, bestcorrected visual acuity; IOP, intraocular pressure

${ }^{*}$ Considering the total number of patients $(n=13)$

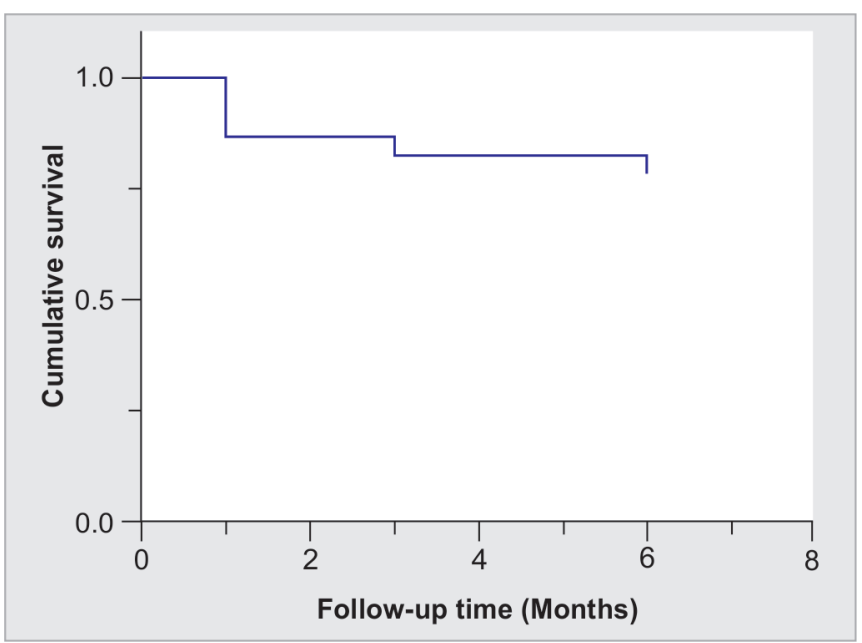

Fig. 2: Kaplan-Meier plot of the probability of survival based on the criteria of failure that was defined as any of the following: IOP out of target range $(5-18 \mathrm{~mm} \mathrm{Hg})$ or $<20 \%$ reduction from baseline for 2 consecutive follow-up visits, additional surgery for glaucoma, or loss of light perception

$-25.3 \pm 21.9$, and $-25.6 \pm 20.9 \%$ at 1,3 , and 6 months of follow-up, respectively (Table 2). Figure 4 displays a scatter diagram showing the pre- and postoperative IOP (a 20\% reduction from baseline IOP was demarcated). For the superior hemifield (Subcyclo), eyes were treated for an average time of $131.7 \pm 26$ seconds (range: 80-150), and for the inferior hemifield (Thermo Cyclo), the treatment had an average laser power of $602.2 \pm 205.3 \mathrm{~mW}$ (range: 100-800) with $25.5 \pm 5.9$ laser applications spots (range: 18-41).

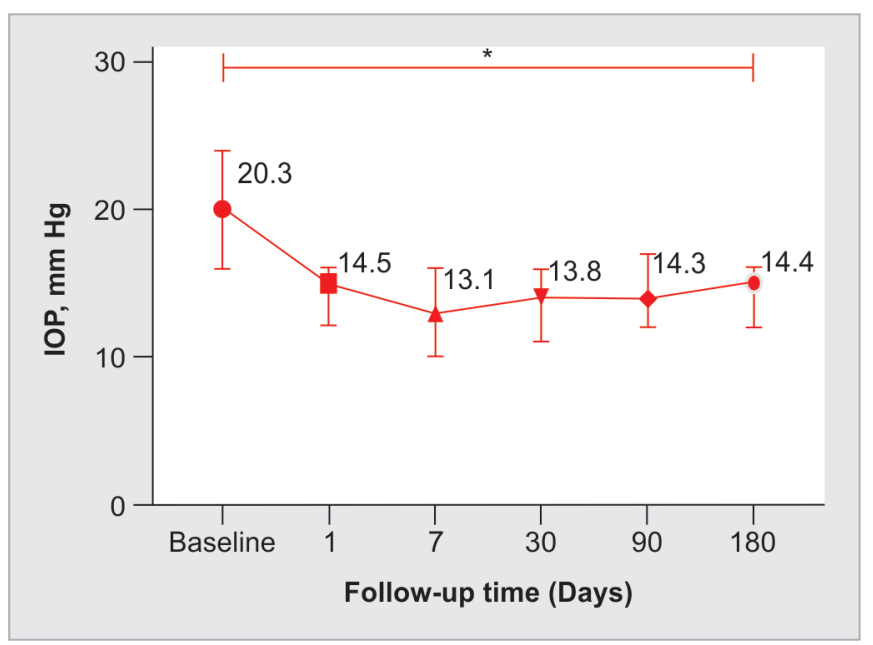

Fig. 3: Line graph depicting the trend in intraocular pressure (IOP; median \pm IQR) in $\mathrm{mm} \mathrm{Hg}$ at baseline and through the follow-up time. *Difference between last from baseline IOP measurement with Wilcoxon rank test $(p<0.0001)$

The mean glaucoma eye drops number after the procedure was $1.2 \pm 1$ (range: $0-4$ ), and the reduction was statistically significant ( $p=0.0024$ ) (Fig. 5). No statistically significant change was found in the mean BCVA compared with preoperative, at 6 months ( $p=$ 0.84 ), with a mean ( \pm SD) final BCVA of $0.76 \pm 0.6$ (range: $0.1-2.1$ ). The distribution of vision changes is shown in Figure $6 ; 78.3 \%$ of the eyes remained within 0.1 LogMAR units of BCVA change, $8.7 \%$ worsened $\geq 0.2$ units, and $13 \%$ improved $\geq 0.2$ LogMAR units. From the two eyes that lost $\geq 2$ lines of BCVA at the last follow-up, one had transient corneal edema with subsequent glaucoma progression, and the other developed vitreous hemorrhage that did not need surgery. No severe complications such as choroidal detachment, phthisis bulbi, persistent hypotony, intraocular inflammation, and loss of light perception were reported.

\section{Discussion}

In our cases with uncontrolled glaucoma, the success rates of Cyclo Mix treatment were 30 and $87 \%$ for complete and qualified successes, respectively, at the last follow-up. The reported success rates by various criteria ranged from 66 to $80 \%$ in approximately 6-12 months, ${ }^{14,15,17-19}$ which are consistent with our results. The small proportion of patients achieving complete success in our study may be due to the variability criteria of success used in the studies mentioned above. These results are promising because our population had advanced glaucoma with different and complex ocular histories. Our modified technique (Cyclo Mix) was intended to provide the benefits from the two strategies, delivering energy in a continuous mode in one hemifield and in a micropulse manner on the other hemifield. Combining these two strategies in one procedure may benefit the outcomes by reducing the aqueous humor production through the selective treatment of the ciliary body epithelium in one area and improve the uveoscleral outflow on the other area. The increased uveoscleral outflow is the mechanism believed to be related to the IOP reduction after an MP TSCPC session. ${ }^{10}$ It is believed that MP TSCPC provides a thermal insult that does not produce tissue damage to the pars plana or ciliary body, thus releasing a biochemical, cellular cascade, which would improve the uveoscleral outflow lowering the IOP. ${ }^{20}$ Even though TSCPC has usually been used in patients with advanced disease and 
Table 2: Evaluated characteristics at consecutive follow-up visits $(n=23)$

\begin{tabular}{lllllll}
\hline Variable & Baseline & Day 1 & Day 7 & Month 1 & Month 3 & Month 6 \\
\hline Eyes, $n$ & 23 & 23 & 23 & 23 & 23 & $14.3 \pm 3.1$ \\
IOP $\bar{x} \pm$ SD $(\mathrm{mm} \mathrm{Hg})$ & $20.3 \pm 5.9$ & $14.5 \pm 5.2$ & $13.1 \pm 6.9$ & $13.8 \pm 4.2$ & $14.4 \pm 3.9$ \\
IOP median (IQR), $(\mathrm{mm} \mathrm{Hg})$ & $20(16-24)$ & $15(12-16)$ & $13(10-16)$ & $14(11-16)$ & $14(12-17)$ & $15(12-16)$ \\
IOP reduction, \% & - & 24.9 & 31.4 & 28.3 & 25.3 & 25.6 \\
Eyes with a $>$ 20\% IOP & - & 52.1 & 69.5 & 69.5 & 62.2 & 62.2 \\
reduction, \% & - & - & - & 47.8 & 52.2 & 30.4 \\
Complete success, \% & - & - & - & $1.0 \pm 1.2$ & $1.0 \pm 1.2$ & $1.2 \pm 1.0$ \\
Number of glaucoma & $2.7 \pm 1.5$ & & & & &
\end{tabular}

$\bar{x}$, mean; SD, standard deviation; IQR, interquartile range; IOP, intraocular pressure

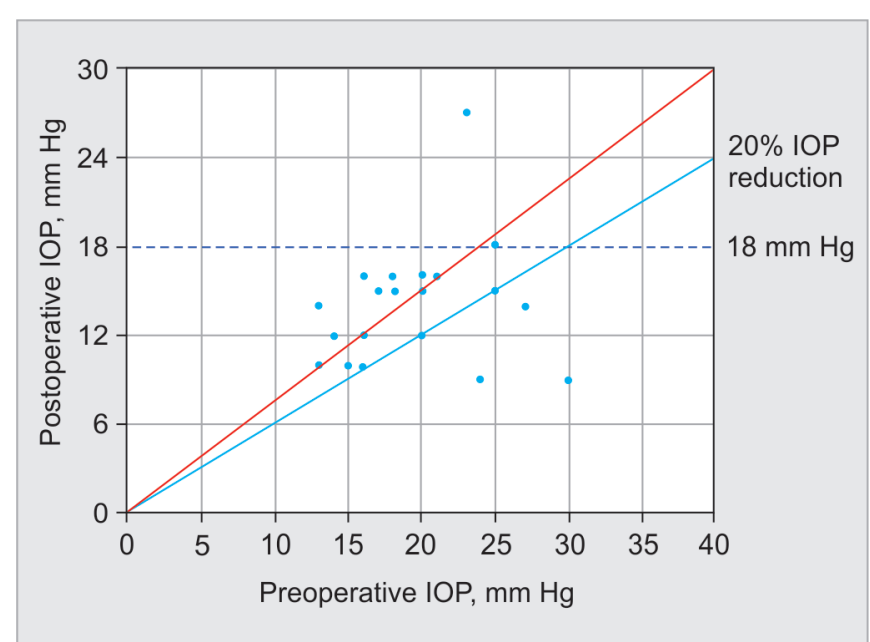

Fig. 4: Scatter plot of preoperative and postoperative intraocular pressure (IOP) after 6 months. Eyes are illustrated as circles. The oblique red line indicates no change of IOP. Circles above the oblique line represent a higher postoperative IOP. Eyes below the line of $18 \mathrm{~mm} \mathrm{Hg}$ or $20 \%$ IOP reduction fulfilled the criteria of qualified success

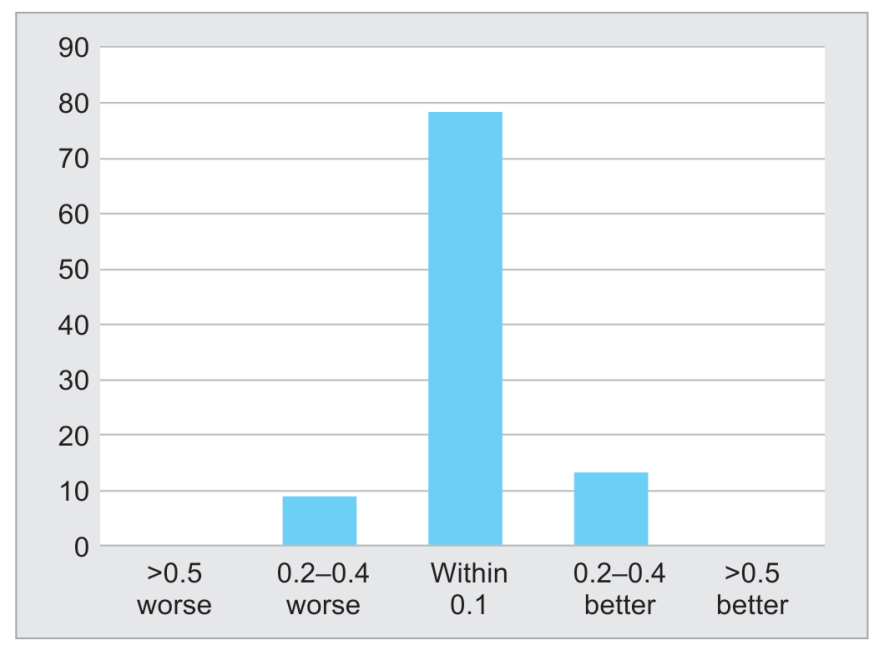

Fig. 6: Distribution of change in best-corrected visual acuity from baseline to 6 months $(n=23)$

insufficient IOP control who also had little visual potential or high risk of visual loss. ${ }^{9}$ All patients in this report had uncontrolled IOP

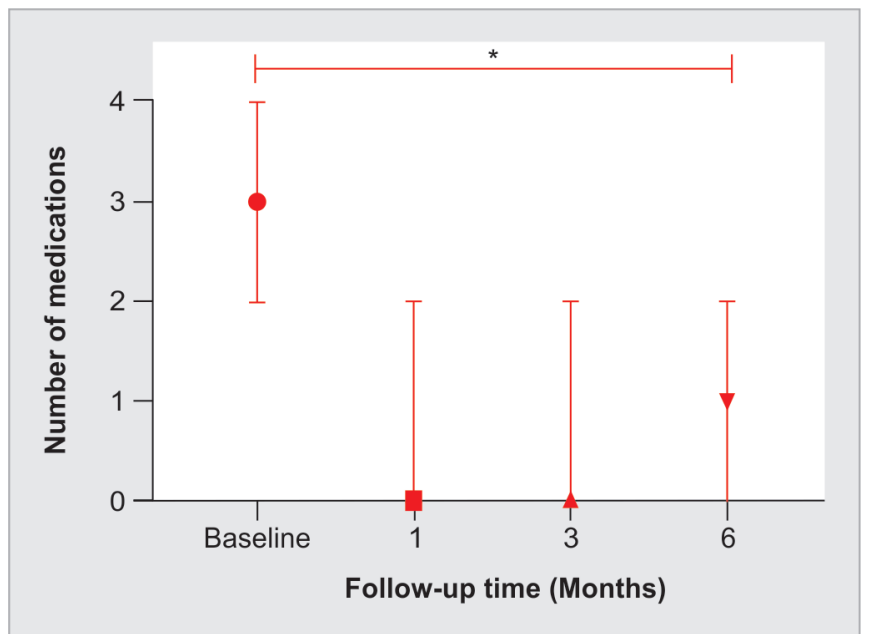

Fig. 5: Number of medications (median $\pm \mathrm{IQR}$ ) at baseline through the last follow-up. *Difference between last from baseline glaucoma medications with paired Student's T-test $(p<0.0001)$

and advanced disease but with visual potential. Visual outcomes in our series are promising; among our 23 cases, only 2 eyes loss $\geq 2$ lines on the follow-up, one case developed a transient vitreous hemorrhage with the subsequent formation of an epiretinal membrane. The second patient developed transitory corneal edema ( 3 weeks) after the procedure; however, vision loss at the last follow-up was due to glaucoma progression. We were not aware of any preexisting corneal pathology (guttate) in our patient; however, previous procedures along, with inflammation and an uncontrolled IOP, may have led to a reduction in endothelial cell count, which could predispose to the development of corneal edema. Our results show that our modified technique is relatively safe and effectively reduces IOP in eyes with uncontrolled glaucoma. Additionally, no severe complications (phthisis or persistent hypotony) were found. Several parameters have been studied in other reports, but few have modified the entire technique; Wong et al. described a modified technique (MP3 plus) using a two-step procedure, combining the conventional continuous wave approach with additional micropulses in an attempt to improve the uveoscleral aqueous outflow. ${ }^{14}$ The treatment success rate achieved in that report was 35.7 and $25.9 \%$ at 6 and 12 months. It is necessary to mention several limitations of this report, including its retrospective design, small sample size, a limited follow-up time, and the absence of a comparative group; thus, more studies are needed to assess the 
real efficacy and mechanism of Cyclo Mix. We believe our Cyclo Mix technique may give an additional effect to the IOP without altering the safety of the standard MP TSCPC procedure. Cyclo Mix seems to be a safe therapy that effectively reduced the number of glaucoma eye drops and IOP levels in eyes with the uncontrolled disease for a period of up to 6 months.

\section{Authorship}

All authors attest that they meet the current ICMJE criteria for authorship.

\section{ACKnOWLedgments}

We gratefully thank the patients for participation in this study.

\section{REFERENCES}

1. Leske MC, Heijl A, Hussein M, et al. Factors for glaucoma progression and the effect of treatment: the early manifest glaucoma trial. Arch Ophthalmol Chic III 1960 2003;121(1):48-56. DOI: 10.1001/ archopht.121.1.48.

2. Tham Y-C, Li X, Wong TY, et al. Global prevalence of glaucoma and projections of glaucoma burden through 2040: a systematic review and meta-analysis. Ophthalmology 2014;121(11):2081-2090. DOI: 10.1016/j.ophtha.2014.05.013.

3. The Advanced Glaucoma Intervention Study (AGIS): 7 The relationship between control of intraocular pressure and visual field deterioration. The AGIS Investigators. Am J Ophthalmol 2000;130(4):429-440. DOI: 10.1016/S0002-9394(00)00538-9.

4. Weinreb RN, Khaw PT. Primary open-angle glaucoma. Lancet Lond Engl 2004;363(9422):1711-1720. DOI: 10.1016/S0140-6736(04) 16257-0.

5. Weve H. Die zyklodiatermie das corpus ciliare bei glaukom. Zentralbl Ophthalmol 1933;29:562-569.

6. Pratesi R. Diode lasers in photomedicine. IEEE J Quantum Electron 1984;20(12):1433-1439. DOI: 10.1109/JQE.1984.1072352.

7. Hennis HL, Stewart WC. Semiconductor diode laser transscleral cyclophotocoagulation in patients with glaucoma. Am J Ophthalmol 1992;113(1):81-85. DOI: 10.1016/S0002-9394(14)75758-7.

8. Maus M, Katz LJ. Choroidal detachment, flat anterior chamber, and hypotony as complications of neodymium: YAG laser cyclophotocoagulation. Ophthalmology 1990;97(1):69-72. DOI: 10.1016/S0161-6420(90)32640-4.
9. Ansari E, Gandhewar J. Long-term efficacy and visual acuity following transscleral diode laser photocoagulation in cases of refractory and non-refractory glaucoma. Eye Lond Engl 2007;21(7):936-940. DOI: 10.1038/sj.eye.6702345.

10. Ma A, Yu SWY, Wong JKW. Micropulse laser for the treatment of glaucoma: a literature review. Surv Ophthalmol 2019;64(4):486-497. DOI: 10.1016/j.survophthal.2019.01.001.

11. Williams AL, Moster MR, Rahmatnejad K, et al. Clinical efficacy and safety profile of micropulse transscleral cyclophotocoagulation in refractory glaucoma. J Glaucoma 2018;27(5):445-449. DOI: 10.1097/ IJG.0000000000000934.

12. Shah P, Bhakta A, Vanner EA, et al. Safety and efficacy of diode laser transscleral cyclophotocoagulation in eyes with good visual acuity. $J$ Glaucoma 2018;27(10):874-879. DOI: 10.1097/IJG.0000000000001057.

13. Emanuel ME, Grover DS, Fellman RL, et al. Micropulse cyclophotocoagulation: initial results in refractory glaucoma. J Glaucoma 2017;26(8):726-729. DOI: 10.1097/IJG.0000000000000715.

14. Wong KYT, Aquino CM, Macasaet AM, et al. Plus: a modified micropulse transscleral cyclophototherapy technique for the treatment of refractory glaucoma. J Glaucoma 2020;29(4):264-270. DOI: 10.1097/IJG.0000000000001443.

15. Keilani C, Benhatchi N, Bensmail D, et al. Comparative effectiveness and tolerance of subliminal subthreshold transscleral cyclophotocoagulation with a duty factor of $25 \%$ versus $31.3 \%$ for advanced glaucoma. J Glaucoma 2020;29(2):97-103. DOI: 10.1097/ IJG.0000000000001409.

16. Heuer D, Barton K, Grehn F, et al. Consensus on definition of success. Text book of guidelines on design and reporting of glaucoma surgical trials. World Glaucoma Association. The Netherlands: Kugler Publications; 2009. pp. 18-24.

17. Aquino MCD, Barton $K$, Tan AMW, et al. Micropulse versus continuous wave transscleral diode cyclophotocoagulation in refractory glaucoma: a randomized exploratory study. Clin Experiment Ophthalmol 2015;43(1):40-46. DOI: 10.1111/ceo.12360.

18. Tan AM, Chockalingam M, Aquino MC, et al. Micropulse transscleral diode laser cyclophotocoagulation in the treatment of refractory glaucoma. Clin Experiment Ophthalmol 2010;38(3):266-272. DOI: 10.1111/j.1442-9071.2010.02238.x.

19. Zaarour K, Abdelmassih $\mathrm{Y}$, Arej N, et al. Outcomes of micropulse transscleral cyclophotocoagulation in uncontrolled glaucoma patients. J Glaucoma 2019;28(3):270-275. DOI: 10.1097/ IJG.0000000000001174.

20. Johnstone MJ, Padilla S, Wen K. Transcleral laser induces aqueous outflow pathway motion \& reorganization. In Coronado, CA 2017 American Glaucoma Society Meeting. 